\title{
De la biologie à la clinique: \\ le décès dû \\ au cancer \\ de la prostate peut-il maintenant être une exception?
}

Fernand Labrie, Leonello Cusan, José Luis Gomez, Bernard Candas, Alain Bélanger, Van Luu-The, Claude Labrie, Jacques Simard

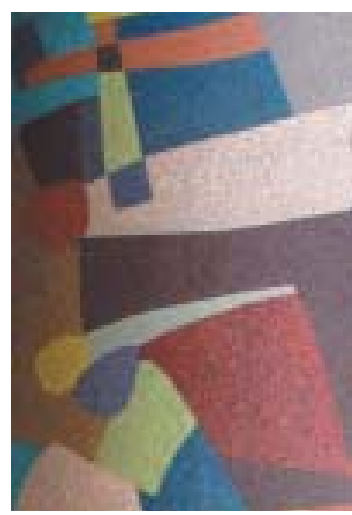

Le cancer de la prostate est devenu le cancer le plus fréquemment diagnostiqué et la seconde cause de décès par cancer chez l'homme [1]. En effet, le cancer de la prostate est diagnostiqué chez un homme sur neuf au cours de sa vie, et cette incidence va en augmentant avec le vieillissement de la population.

Par ailleurs, parmi tous les cancers hormonosensibles, le cancer de la prostate est celui qui est le plus sensible aux hormones. Ainsi, au stade métastatique, le seul traitement efficace du cancer de la prostate est le traitement hormonal. Par ailleurs, dans le cancer cliniquement localisé, le traitement hormonal a montré un succès remarquable, sous forme d'un contrôle à long terme ou même d'une guérison probable dans $90 \%$ des cas [2]

\section{Historique}

Jusqu'en 1980 [3, 4], le traitement standard du cancer de la prostate au stade avancé a été limité au blocage des androgènes testiculaires par castration chirurgicale ou par administration de fortes doses d'œstrogènes tel 
qu'il avait été initié en 1941 [4] (Figure 1). On ne dispose pas de données obtenues à partir d'études randomisées qui démontrent que ces deux traitements prolongent la vie. Par ailleurs, l'œstrogénothérapie entraînait des complications cardiovasculaires tellement importantes que leur usage est devenu fort limité. Enfin, la castration chirurgicale rencontrait des objections d'ordre psychologique chez la plupart des patients.

Après nos premières observations chez l'animal démontrant que le traitement chronique avec des agonistes de la LH-RH (luteinizing hormone-releasing hormone) bloquait par effet antigonadotrope la sécrétion des androgènes testiculaires, entraînant ainsi l'atrophie secondaire de la prostate, on a pu rapidement démontrer chez l'homme que les agonistes de la LH-RH pouvaient remplacer la castration chirurgicale et l'administration d'œstrogènes dans le traitement du cancer de la prostate [5]. De fait, l'homme est l'espèce chez laquelle la castration médicale est la plus facile à obtenir avec les agonistes de la LH-RH. L'excellente tolérance à ce traitement a d'ailleurs été l'élément majeur ayant permis d'effectuer la première série d'études démontrant un effet bénéfique très important du blocage hormonal sur la survie dans le cancer localisé de la prostate [5-11].

\section{Intracrinologie}

\section{Mécanismes de l'intracrinologie}

Une découverte d'importance majeure dans le domaine de la physiologie des stéroïdes sexuels est celle démontrant que l'homme, de même que les autres primates supérieurs, sont les seuls parmi les espèces animales à posséder des glandes surrénales sécrétant des quantités importantes de précurseurs stéroïdiens inactifs, déhydroépiandrostérone (DHEA) et son sulfate (DHEA-S), qui sont convertis en androgènes et œstrogènes actifs dans les tissus périphériques cibles (Figures 2 et 3 ). Les niveaux élevés de DHEA et de DHEA-S dans la circulation fournissent une quantité importante de substrats ou de précurseurs qui peuvent être transformés en androgènes et en œstrogènes actifs dans les tissus périphériques. Le terme «intracrinologie»a été inventé en 1988 [11], afin de décrire la biosynthèse des stéroïdes actifs dans les tissus périphériques cibles où ces stéroïdes exercent leur action dans les cellules mêmes où leur synthèse a lieu (Figure 2). Ces stéroïdes ne diffusent qu'en très faible quantité dans l'espace extracellulaire et dans la circulation générale [12]. Les connaissances dans le domaine de l'intracrinologie ont fait un progrès majeur après l'élucidation de la structure de la plupart des ADN complémentaires et des gènes codant pour les enzymes de la stéroïdogenèse responsables de la transformation de DHEA et DHEA-S en androgènes et en œstrogènes dans les tissus périphériques $[12,13]$ (Figure 3). Il est en effet remarquable de constater que l'homme, qui possède par ailleurs un système endocrinien très sophistiqué, compte en grande partie sur la formation des stéroïdes sexuels dans les tissus périphériques pour assurer le fonctionnement normal de l'organisme. Ainsi, chez l'homme et les primates, les stéroïdes sexuels sont formés en grande partie dans les tissus périphériques à partir de la DHEA, ce qui confère à ces tissus une autonomie grâce à laquelle ils peuvent ajuster la formation et le métabolisme des stéroïdes sexuels selon les besoins locaux, ce qui est l'essence même de l'intracrinologie [11-15]. Cette absence de dilution des hormones dans le compartiment extracellulaire et dans la circulation générale permet de réduire au minimum la quantité d'hormones requises pour exercer un contrôle efficace dans les tissus cibles dépendants de ces hormones.
Figure 1. Historique des étapes principales de l'évolution du traitement hormonal du cancer de la prostate de 1941 à 2003. LH-RH: luteinizing hormone-releasing hormone. 
Il est important de mentionner que près de $40 \%$ de tous les cancers (sein, prostate, ovaire, utérus) dépendent des stéroïdes sexuels et sont donc des candidats de premier choix pour des approches thérapeutiques fondées sur le contrôle de l'activité intracrine. Ce champ de l'endocrinologie a d'ailleurs déjà eu des répercussions très importantes dans le traitement des deux cancers les plus fréquents, respectivement chez l'homme et chez la femme, ceux de la prostate et du sein. L'intracrinologie offre des voies fort prometteuses dans le domaine des inhibiteurs des enzymes de la stéroïdogenèse. Ces connaissances nouvelles sont une incitation pour mettre au point des antiœstrogènes et des antiandrogènes, ainsi que des inhibiteurs plus puissants de la stéroïdogenèse qui pourront contrôler de plus en plus efficacement et spécifiquement la formation ou l'action des stéroïdes sexuels formés localement dans les tissus intracrines.

\section{Double source d'androgènes}

Bien que la castration chirurgicale [3] ou l'administration d'un agoniste de la LH-RH [4] entraîne une diminution de 80 à $90 \%$ des niveaux circulants de testostérone [4, 13], un effet beaucoup plus faible est observé sur le paramètre directement responsable de la stimulation de la cellule prostatique normale ou cancéreuse, à savoir la concentration intraprostatique de dihydrotestostérone (DHT), l'androgène le plus puissant. De fait, les niveaux intraprostatiques de DHT sont diminués de seulement 50 à $70 \%$ après castration médicale ou chirurgicale [13] (Figure 4).

S'ajoutant aux androgènes d'origine testiculaire, les androgènes fabriqués localement dans la prostate, à partir de la DHEA, exercent essentiellement leur action localement dans les cellules mêmes, sites de leur formation. Tel que mentionné ci-dessus, la DHT ainsi formée dans la prostate ne diffuse pas à l'extérieur de la cellule avant d'avoir été inactivée ou métabolisée en dérivés glucuronides, qui sont hydrosolubles et diffusent facilement dans la circulation. Contrairement à la conviction antérieure qui voulait que les testicules soient res- ponsables de 90 à $95 \%$ de la production totale d'androgènes chez l'homme, tel que cela était suggéré par la simple mesure des niveaux circulants de testostérone (Figure 4), il est maintenant bien démontré que le tissu prostatique possède tous les mécanismes enzymatiques lui permettant de transformer localement les précurseurs stéroïdiens inactifs DHEA-S et DHEA en testostérone et en DHT. Ainsi, bien que 50 à $70 \%$ des androgènes soient éliminés par la castration médicale ou chirurgicale, la seule méthode pouvant neutraliser l'action des androgènes d'origine surrénalienne formés dans la prostate est l'ajout d'un antiandrogène pur, qui prévient l'interaction de la testostérone et de la DHT avec le récepteur des androgènes [16-18] (Figure 5).

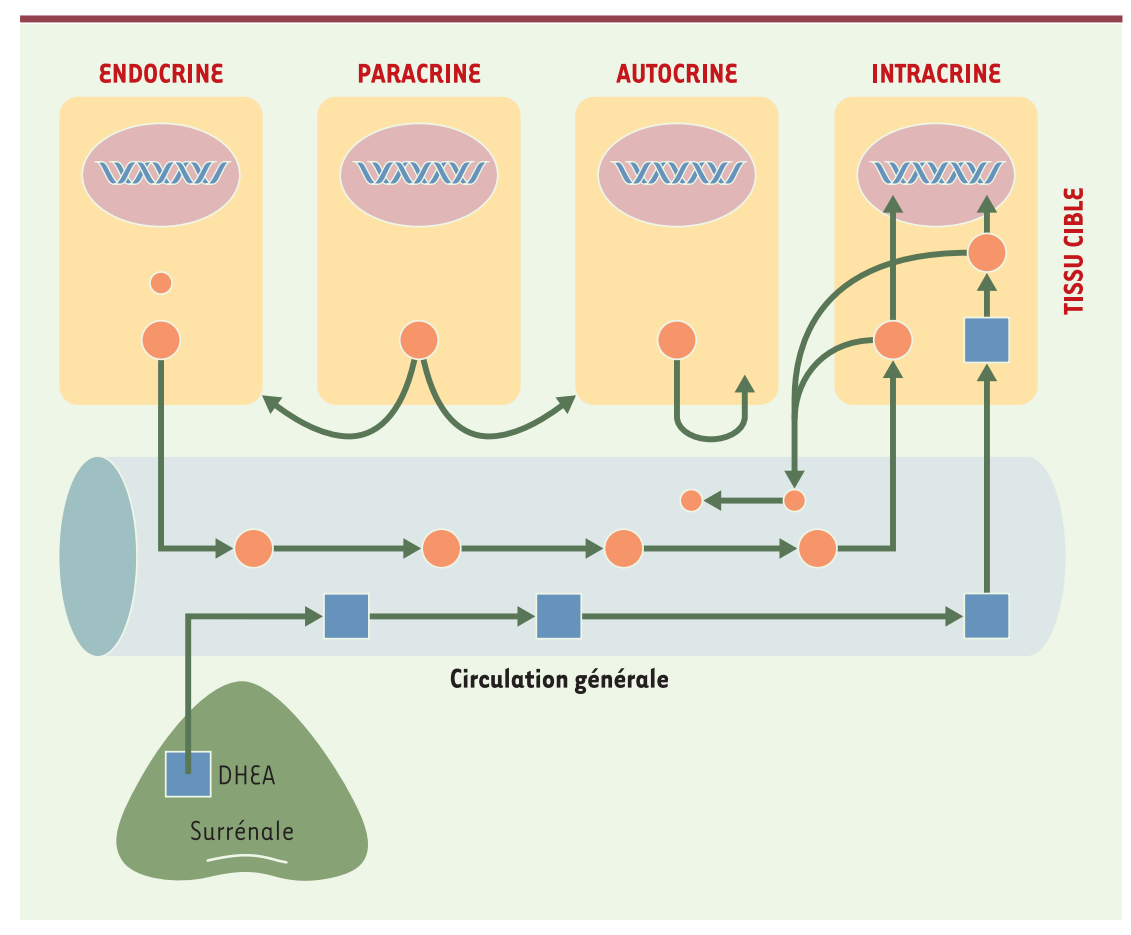

Figure 2. Schéma des sécrétions endocrine, paracrine, autocrine et intracrine. De façon classique, l'activité endocrine se réfère aux hormones synthétisées par des glandes spécialisées, comme les testicules. Ces hormones sont ensuite libérées dans la circulation générale et transportées vers les cellules cibles situées à distance. Par ailleurs, les hormones libérées d'une cellule peuvent influencer l'activité des cellules voisines, ce qui constitue l'activité paracrine, alors que d'autres hormones libérées d'une cellule peuvent exercer leur action positive ou négative sur la cellule même qui les a sécrétées, ce qui constitue une activité autocrine. L'activité intracrine décrit la formation d'hormones actives à partir du précurseur déhydroépiandrostérone (DHEA) synthétisé au niveau des surrénales. Ces hormones formées localement exercent leur action à l'intérieur même des cellules où elles ont été formées, sans libération significative dans le compartiment extracellulaire et la circulation générale. Par ailleurs, ces stéroïdes actifs sont inactivés dans les mêmes cellules sous forme de dérivés glucuronides et sulfates qui diffusent facilement dans la circulation pour être éliminées, surtout par le foie et par le rein [13, 42]. 


\section{Blocage androgénique combiné}

Après l'obtention de nos données initiales [16], une série d'études prospectives et randomisées effectuées par plusieurs groupes [19-24] ont confirmé une prolongation de la vie après blocage androgénique combiné utilisant l'ajout d'un antiandrogène pur à la castration médicale ou chirurgicale chez des patients ayant un cancer avancé de la prostate. Bien que les données cliniques ne soient pas encore disponibles pour le bicalutamide (Casodex ${ }^{\circledR}$ ), celles concernant les deux antiandrogènes purs, flutamide $\left(\right.$ Eulexin $\left.^{\circledR}\right)$ et nilutamide (Nilandron ${ }^{\circledR}$ ), ont démontré, dans des études prospectives et randomisées, que la vie était prolongée, tout en augmentant le nombre de réponses complètes et partielles au traitement. La progression de la maladie est également retardée et le contrôle de la douleur est obtenu plus rapidement chez un plus grand nombre de patients, ce qui augmente leur qualité de vie [19-24]. Les données mentionnées ci-dessus devraient mettre fin à la controverse concernant les bénéfices du blocage androgénique. En effet, l'ajout d'un antiandrogène pur à la castration augmente la survie de 3 à 6 mois de façon générale et de 6 à 12 mois lorsque l'on considère seulement les décès dus au cancer de la prostate. Pour la population totale d'hommes vivant en Amérique du Nord, où I'on estime que 3 millions d'entre eux vont décéder du cancer de la prostate si aucun changement ne survient, 6 mois additionnels de vie correspondent à l'ajout de 1,5 million d'années-homme.

\section{Importance du diagnostic précoce et donc du dépistage}

Malgré les progrès significatifs réalisés dans le traitement du cancer de la prostate au stade avancé [13, 16, 19-24], il est bien reconnu que la seule possibilité d'obtenir une diminution importante des décès dus au cancer de la prostate est le traitement à une stade cliniquement localisé. Face à ces constatations, nous avons débuté, en 1988, dans la région de Québec, un

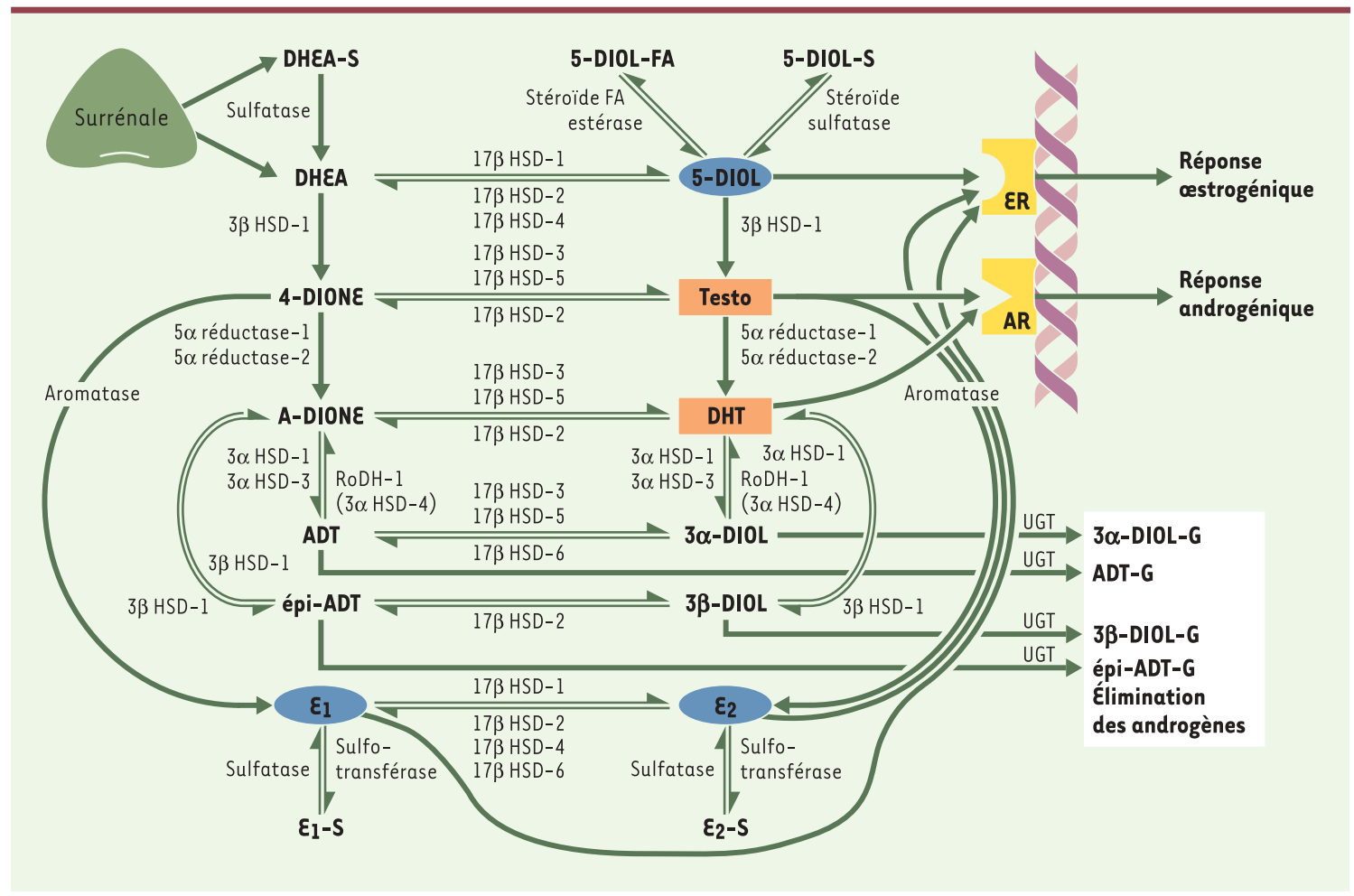

Figure 3. Enzymes de la stéroïdogenèse et de l'inactivation des stéroïdes sexuels dans les tissus intracrines périphériques humains. La surrénale sécrète des quantités importantes de déhydroépiandrostérone (DHEA) et de son sulfate (DHEA-S). L'enzyme $3 \beta$ hydroxystéroïde déshydrogénase (3ßHSD) transforme la DHEA en androstènedione (4-dione), qui peut être convertie en testostérone (Testo) par la $17 \beta$-hydroxystéroïde déshydrogénase ou en œstrone ( $\varepsilon 1)$ par l'aromatase. La testostérone est ensuite transformée en dihydrotestostérone (DHT), l'androgène le plus actif, par la $5 \alpha$-réductase. Les stéroïdes actifs interagissent avec leurs récepteurs spécifiques, récepteurs des œstrogènes $(\varepsilon R)$, récepteurs des androgènes $(A R)$, pour être ensuite inactivés, surtout par les glucuronyltransférases (UGT) et par les sulfotransférases. 
programme de dépistage du cancer de la prostate afin d'évaluer l'influence sur la survie d'un diagnostic précoce et d'un traitement appliqué au stade localisé de la maladie. Grâce à la participation des hommes âgés de 45 à 80 ans de la région métropolitaine de Québec, nous avons pu réaliser la première étude randomisée et prospective au monde sur ce sujet.

De novembre 1988 à décembre 1999, 7348 hommes ont subi les tests annuels de dépistage au Centre hospitalier de l'université Laval. Ces tests incluaient le dosage de l'APS (antigène prostatique spécifique) et un toucher rectal. Depuis 1993, I'APS seul a été utilisé lors des visites de suivi. En effet, nous avons constaté avant cette date qu'environ 5000 touchers rectaux étaient requis pour découvrir un cas de cancer de la prostate lorsque l'APS était inférieur à $3 \mathrm{ng} / \mathrm{ml}$. Pendant la période allant du 15 novembre 1988 au 31 décembre 1999, le taux de décès dus au cancer de la prostate a diminué de $64 \%$ (soit trois fois moins de décès) dans le groupe d'hommes dépistés au CHUL comparativement aux hommes du groupe témoin n'ayant pas été dépistés au CHUL. Ce résultat démontre bien l'efficacité du dépistage et du traitement précoces. II est important de préciser que dans le groupe des hommes dépistés, seulement 10 décès dus au cancer de la prostate sont survenus, comparativement à un nombre trois fois plus élevé chez les hommes du groupe témoin.

Ces treize années d'efforts consacrés à ce programme de recherche, incluant plus de 60000 visites annuelles au CHUL, ont clairement démontré les faits suivants:

- le dépistage annuel par le dosage de l'APS permet de diagnostiquer $99 \%$ des cancers de la prostate à un stade précoce [25-27];

- la technique de dépistage est en général facilement acceptée par la population. En effet, il s'agit d'une simple prise de sang pour mesure de I'APS au laboratoire et d'un toucher rectal à la première visite, alors que pour les visites annuelles de suivi, la mesure d'APS suffit;

- ces cancers diagnostiqués par dépistage sont généralement guérissables par les traitements actuellement disponibles [2, 26, 28, 29]. En effet, le dépistage mis au point au CHUL a permis de diminuer les décès dus au cancer de la prostate d'environ 3 fois, ce qui est un résultat unique dans le domaine du cancer (dernière analyse des données obtenues entre novembre 1988 et décembre 1999). Bien entendu, une fois le cancer de la prostate diagnostiqué au stade précoce par dépistage, un traitement par chirurgie, radiothérapie, brachythérapie et/ou blocage androgénique doit être appliqué sans délai. Il est important d'indiquer que parmi les 10 patients décédés, il s'agissait, pour 6 d'entre eux, d'un cancer découvert à un stade déjà avancé lors de la première visite. Les quatre autres patients diagnostiqués lors des visites de suivi n'ont pas reçu le traitement immédiat et continu recommandé par notre équipe, ou le traitement suivi dans un autre milieu n'est pas connu de nous. De fait, comme le démontrent clairement nos données, si le dépistage du cancer de la prostate débute à l'âge de 50 ans, et en accord avec les recommandations de la Société américaine du cancer et de la Société américaine d'urologie [30], les décès dus au cancer de la prostate devraient maintenant être une exception. Toutes les études internationales prospectives et randomisées effectuées sur une grande échelle

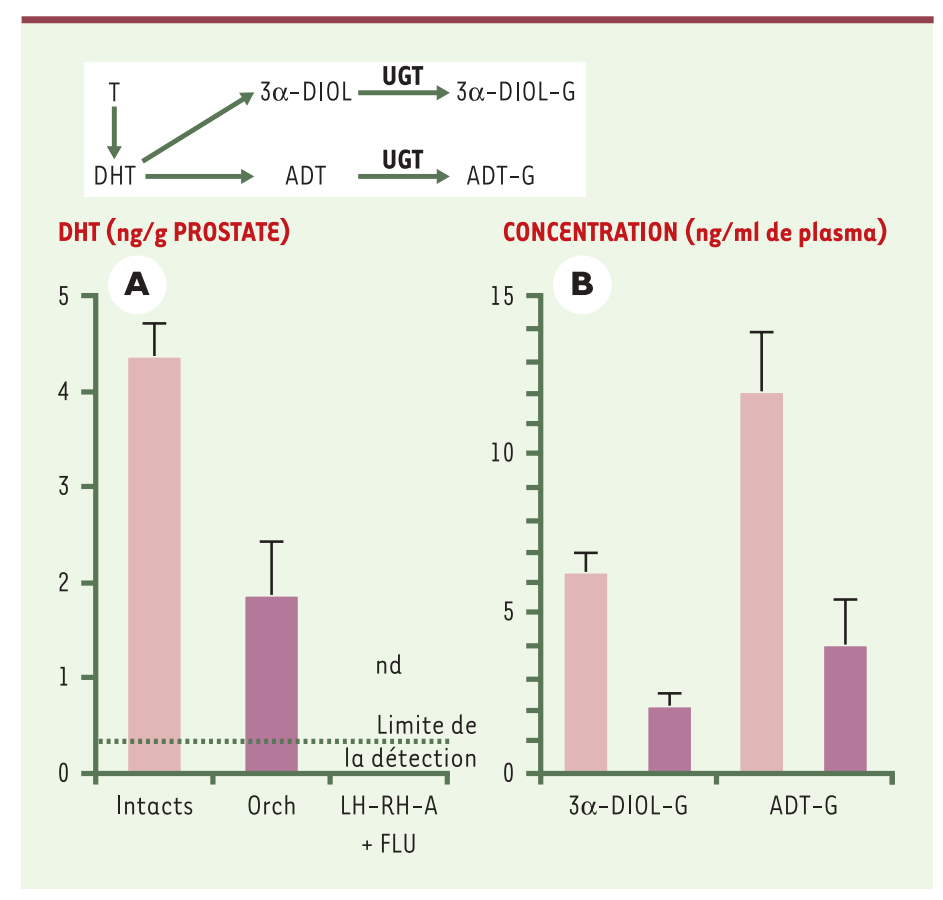

Figure 4. Effet de l'orchidectomie et du blocage androgénique combiné (addition d'un antiandrogène pur, le flutamide, à l'orchidectomie ou au traitement avec un agoniste de la $\mathrm{LH}-\mathrm{RH}$ ) sur la concentration de dihydotestostérone (DHT) dans le tissu prostatique cancéreux humain. A. Du tissu prostatique cancéreux a été obtenu chez trois groupes de patients, un groupe de patients n'ayant reçu aucun traitement hormonal (intacts), un deuxième groupe de patients (orch) chez lesquels la sécrétion de testostérone par les testicules a été éliminée par castration chirurgicale ou par traitement avec un agoniste de la LH-RH, un troisième groupe de patients ayant reçu, outre la castration médicale, un antiandrogène pur, le flutamide (FLU) (nd, non détecté). B. L'effet sur la concentration intratumorale de DHT, les taux sanguins des deux principaux métabolites des androgènes, l'androstane-3 $\alpha, 17 \beta$-diol-glucuronide (3 $\alpha$-diol-G) et l'androstérone glucuronide (ADT-G) ont été déterminés. Cette figure rappelle également (en haut) la transformation de la testostérone $(T$ ) en dihydrotestostérone (DHT). La DHT est par la suite transformée en androstane$3 \alpha, 17 \beta$-diol (3 $\alpha$-diol) ou androstérone (ADT). Ces métabolites sont ensuite conjugués par les glucuronyltransférases (UGT) pour former le $3 \alpha$-diol-glucuronide et I'ADT glucuronide. 
sont venues confirmer nos résultats. Ainsi, une étude finlandaise effectuée chez 20176 participants confirment que l'APS seul permet un dépistage efficace du cancer de la prostate [31]. Ces résultats viennent ainsi confirmer ceux que nous avons déjà obtenus en 1992

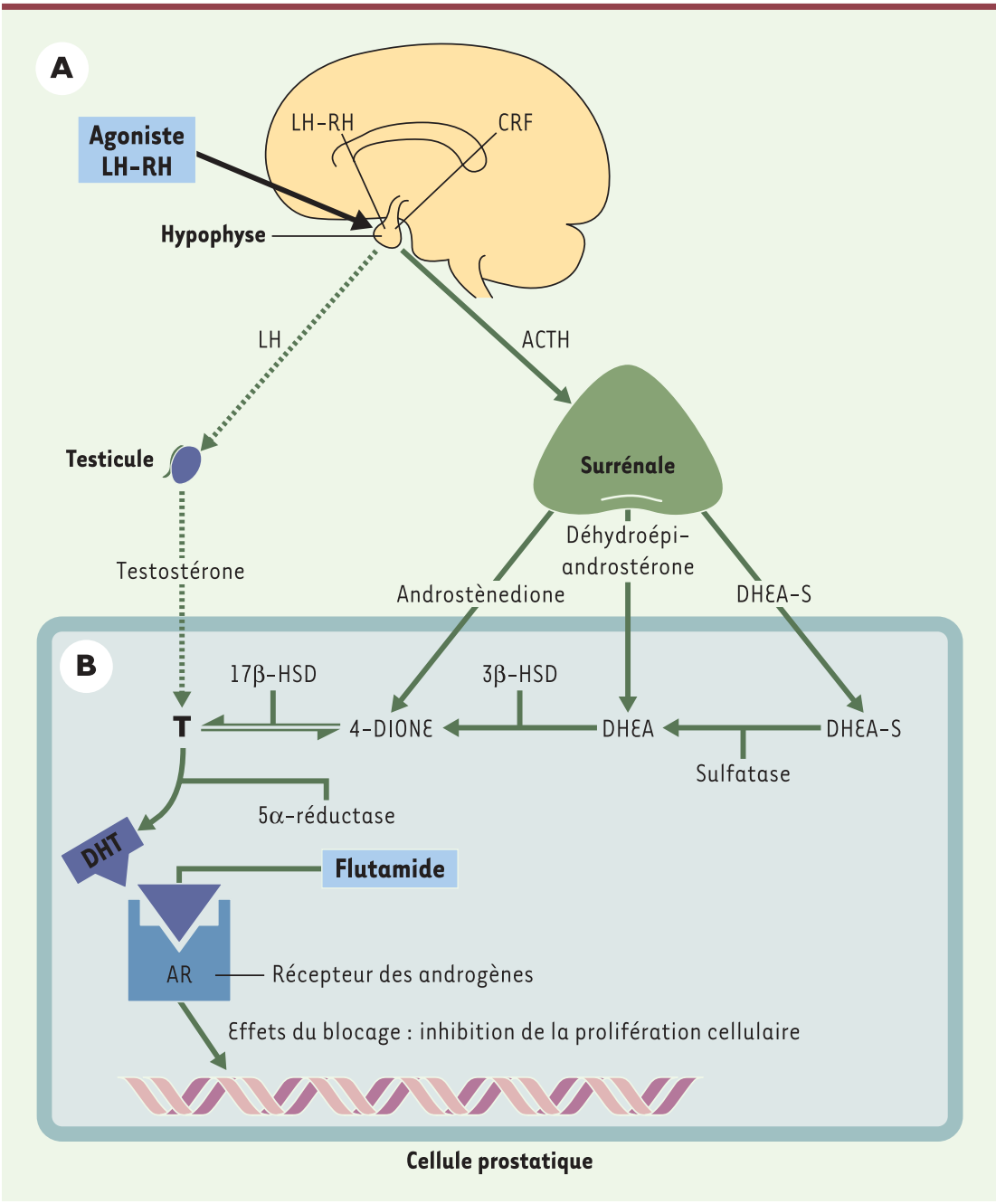

Figure 5. Représentation schématique de l'effet du blocage androgénique combiné avec un agoniste de la LH-RH et un antiandrogène pur (flutamide) sur la croissance du cancer prostatique. Alors que la castration chimique avec un agoniste de la LH-RH élimine la sécrétion de testostérone par les testicules, les surrénales sécrètent des quantités importantes de déhydroépiandrostérone (DHEA) et de DHEA-sulfate (DHEA-S), de même qu'une certaine quantité d'androstènedione (4-dione). La prostate humaine contient les enzymes permettant de transformer la DHEA-S en DHEA puis en 4-dione. Ce dernier stéroïde est transformé par la $17 \beta$-hydroxystéroïde déshydrogénase (17 $\beta$-HSD) en testostérone (T) puis en dihydrotestostérone (DHT). La façon la plus efficace de bloquer l'action de la DHT est d'utiliser un antiandrogène pur, comme le flutamide, qui bloque l'interaction de la DHT avec le récepteur des androgènes (AR). Cette élimination de la sécrétion des androgènes testiculaires associée au blocage de l'action des androgènes d'origine surrénalienne permet un blocage plus complet des androgènes stimulateurs de la croissance du cancer prostatique. Cette stratégie est appelée blocage androgénique combiné. II s'agit du premier traitement démontré comme prolongeant la vie dans le cancer de la prostate [16, 19-24]. accessible sur une grande échelle dans la population.

\section{Grande efficacité} du traitement hormonal dans le cancer au stade localisé

Diminution du taux de décès par blocage androgénique

Malgré les progrès importants réalisés dans le traitement hormonal du cancer de la prostate au stade avancé, il est bien reconnu que la seule façon d'avoir un impact majeur sur la survie est le traitement du cancer au stade localisé. De fait, au cours des dernières années, six études prospectives et randomisées sont venues démontrer un impact majeur du blocage androgénique sur la prolongation de la vie dans le cancer localisé de la prostate (Tableau 1). Ainsi, en considérant les décès dus au cancer de la prostate à 5 années de suivi après le début du traitement, la diminution du taux de décès se situe entre $37 \%$ et $81 \%$ dans les différentes études, ce qui est un résultat remarquable dans le domaine du cancer. Ainsi, la première étude à être publiée, celle de Bolla et al. en 1997 [5] montre une diminution de $77 \%$ des décès dus au cancer de la prostate. Un autre résultat remarquable est celui de l'étude de Messing et al. en 1999 [9], qui montre une diminution des décès dus au cancer de la prostate de $81 \%$ chez les patients ayant des métastases ganglionnaires pelviennes découvertes lors de la chirurgie, et qui ont été randomisés entre le blocage androgénique immédiat ou retardé.

Toutes les études publiées (Tableau I) démontrent clairement un avantage marqué sur la survie du traitement hormonal administré immédiatement lors du diagnostic. Ces résultats éliminent 
les arguments antérieurs qui ont malheureusement retardé l'application du dépistage et du diagnostic précoce, sous prétexte qu'aucune étude n'avait jusqu'alors démontré les avantages sur la survie d'un traitement débuté à un stade précoce de la maladie. Un point important à souligner est qu'aucune étude visant à démontrer un effet positif du traitement précoce sur la survie n'avait été effectuée.

Il faut maintenant reconnaître que le traitement précoce du cancer de la prostate, comme celui de tout autre cancer, est la seule façon de pouvoir guérir la maladie et d'avoir un impact majeur sur la survie. De fait, l'attente de métastases osseuses avant de débuter le traitement signifie qu'il est alors trop tard pour envisager la guérison et que le seul espoir qui demeure est de prolonger la vie de quelques mois, ce qui est loin d'être satisfaisant. En effet, compte tenu de l'état actuel des connaissances, les traitements hormonaux disponibles permettent d'obtenir des résultats impressionnants sur la durée de vie, et même obtenir une guérison dans la majorité des cas diagnostiqués et traités au stade cliniquement localisé.

\section{Possibilité de guérison avec le blocage androgénique} combiné

Il est très important d'indiquer que le blocage androgénique combiné est remarquablement efficace dans le cancer localisé de la prostate. En effet, chez 26 patients qui ont reçu un antiandrogène pur (flutamide) en association avec un agoniste de la LH-RH pendant une période allant jusqu'à 12 années (médiane = 7,1 années), la première augmentation de l'APS est survenue chez seulement 1 patient après 7 années et 4 mois de traitement [37]. Les effets secondaires du blocage androgénique combiné sont généralement bien tolérés. Ces effets secondaires incluent les bouffées de chaleur qui durent habituellement quelques mois au début du traitement, la perte d'appétit sexuel et l'impuissance sexuelle $[13,19]$. Les données résumées ici indiquent que le blocage androgénique combiné est très efficace dans le traitement du cancer de la prostate au stade localisé, son efficacité se comparant avantageusement à celle de la chirurgie ou de la radiothérapie seule [37].

\section{L'efficacité du traitement hormonal requiert un traitement continu et à long terme}

La Figure 6 illustre l'effet de la durée du blocage androgénique combiné sur le succès du traitement hormonal. Lorsque le traitement a été administré pendant seulement une année chez un groupe de 11 patients porteurs de cancer au stade B2, une augmentation de l'APS a été observée chez tous les patients moins d'une année après l'arrêt du traitement, ce qui signifie que le cancer était encore présent malgré un APS non détectable. En revanche, chez 20 patients porteurs de cancer de la prostate au même stade et traités pendant une durée moyenne de 6,5 années ou plus, I'APS est demeuré non détectable chez $90 \%$ d'entre eux pendant au moins 5 ans après avoir cessé le traitement hormonal, ce qui suggère fortement une guérison. Fait important à noter, I'APS est redevenu non détectable chez tous les patients après reprise du traitement hormonal.

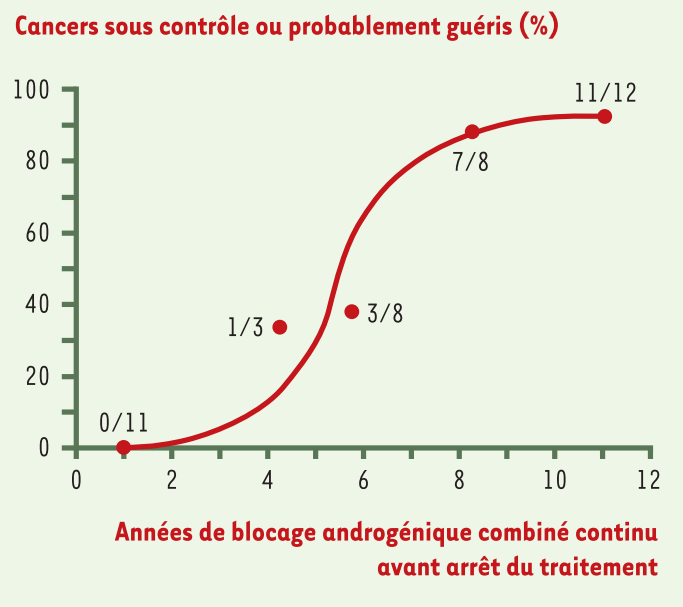

Figure 6. Effet de la durée du blocage androgénique combiné sur le contrôle à long terme ou la guérison probable du cancer localisé de la prostate. Une réponse est considérée comme positive (cancer sous contrôle ou guérison probable) lorsque l'antigène prostatique spécifique (APS) demeure non détectable pendant 5 ans ou plus après arrêt du traitement. Ainsi, lorsque le traitement est administré pendant seulement un an, une augmentation de l'APS a été observée dans tous les cas, ce qui indique un succès nul chez les 11 patients ainsi traités pendant cette période de temps $(0 / 11)$. Par ailleurs, lorsque le traitement a duré plus de 6,5 années, $90 \%$ des patients ont montré un cancer sous contrôle ou une guérison probable. Ainsi, 7 des 8 patients traités pendant huit ans ont montré une telle réponse, alors que 11 sur 12 traités pendant 11 ans présentent la même réponse, soit une guérison probable. Par ailleurs, le traitement d'une durée intermédiaire (4,5 années) a montré une réponse dans 1 cas sur 3 , alors qu'une réponse considérée comme positive est observée dans seulement 3 cas sur 8 quand le traitement dure un peu moins de 6 ans. Ces données démontrent qu'un traitement continu et à long terme du cancer de la prostate localisé avec le blocage androgénique combiné offre un espoir de guérison probable dans $90 \%$ des cas, si le traitement est d'une durée de plus de 6,5 années [2]. 
De même, nous avons noté les bénéfices additionnels d'une durée de traitement de 10,5 mois comparativement à 3 mois chez des patients qui avaient reçu une radiothérapie [38]. De façon similaire, il a été démontré qu'avec le tamoxifène administré comme thérapie adjuvante à la chirurgie chez des patientes souffrant de cancer du sein, les effets observés étaient supérieurs après une période de 5 ans de traitement comparativement à une durée de traitement de 2 ans [39], ce qui supporte la recommandation d'administrer le blocage androgénique combiné pendant une période de plusieurs années, probablement 6 années au minimum.

\section{Conclusions}

Les données résumées ci-dessus et décrites plus en détail dans d'autres publications nous indiquent qu'avec les méthodes actuelles de dépistage et l'application des traitements aujourd'hui disponibles, le décès du au cancer de la prostate devrait être une exception. Comme nous l'avons mentionné plus haut, on estime que 3 millions d'hommes vivant actuellement en Amérique du Nord décéderont du cancer de la prostate si les taux de décès dus à cette maladie demeurent inchangés. La diminution de $64 \%$ du taux de décès dans l'étude de dépistage effectuée à Québec correspondrait à sauver la vie de 2 millions d'hommes si la même approche était appliquée en Amérique du Nord. Bien sûr, le traitement hormonal comporte des effets secondaires, mais ces derniers doivent être mis en perspective avec les effets secondaires associés à d'autres traitements comme la chimiothérapie. Ces effets secondaires prennent également moins d'importance lorsque l'on considère l'efficacité exceptionnelle du traitement hormonal combiné dans le cancer localisé de la prostate, où la guérison peut possiblement être obtenue dans $90 \%$ des cas. $\diamond$

\section{SUMMARY}

From the gene to the clinic: prostate cancer death can now be an exception?

The most significant discovery of the second half of the $x x^{\text {th }}$ century in the field of prostate cancer therapy is probably the observation that the human prostate, as well as many other peripheral human tissues, synthesize locally an important amount of androgens from the inactive steroid precursors dehydroepiandrosterone (DHEA) and its sulfate DHEA-S. In parallel with these observations, two important discoveries also made by our group are applied in the clinic worlwide, namely the use of LHRH (luteininizing hormone-releasing hormone) agonists to completely block testicular androgens, while, simultaneously, the androgens made locally in the prostate from DHEA are blocked in their access to the androgen receptor by a pure antiandrogen of the class of flutamide. This treatment, called combined androgen blockade, has been the first treatment demonstrated to prolong life in prostate cancer. While the first studies were performed in patients with advanced and metastatic disease, our recent data indicate a remarkable level of efficacy of the same treatment applied to localized prostate cancer, namely a $90 \%$ possibility of cure. However, in order to be able to treat localized prostate cancer, early diagnosis must be achieved. In the first large-scale randomized study of prostate cancer screening, we have demonstrated that $99 \%$ of prostate cancers can be diagnosed at the localized or potentially curable stage, using simple annual measurement of PSA (prostatic specific antigen). Today's data show that with the simple application of the available diagnostic and therapeutic tools, death from prostate cancer should be an exception. $\diamond$

\begin{tabular}{|c|c|}
\hline Études & Bénéfices \\
\hline हORTC [5] & $\begin{array}{l}\text { Diminution de } 77 \% \text { des décès dus au } \\
\text { cancer de la prostate }(p=0,01)\end{array}$ \\
\hline RTOG [6] & $\begin{array}{l}\text { Diminution de } 37 \% \text { des décès chez les patients } \\
\text { ayant un score de Gleason de } 8 \text { à } 10(p=0,03)\end{array}$ \\
\hline Étude de dépistage de Québec [8] & $\begin{array}{l}\text { Diminution de } 64 \% \text { des décès dus au } \\
\text { cancer de la prostate }(p=0,0002)\end{array}$ \\
\hline$[7]$ & $\begin{array}{l}\text { Diminution de } 81 \% \text { des décès dus au } \\
\text { cancer de la prostate }(p=0,001)\end{array}$ \\
\hline [9] & $\begin{array}{l}\text { Diminution de } 39 \% \text { des décès dus au } \\
\text { cancer de la prostate }(p=0,06)\end{array}$ \\
\hline$[10]$ & $\begin{array}{l}\text { Diminution de } 59 \% \text { des décès chez les patients } \\
\text { ayant un score de Gleason de } 8 \text { à } 10(p=0,007)\end{array}$ \\
\hline
\end{tabular}

Tableau I. Études randomisées montrant une diminution des décès dus au cancer de la prostate par blocage androgénique.

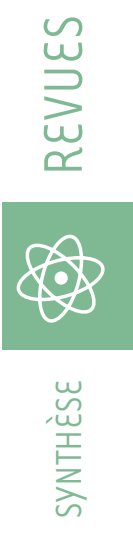




\section{RÉFÉRENCES}

1. Jemal A, Murray T, Samuels A, Ghafoor A, Ward $\varepsilon$, Thun MJ. Cancer statistics 2003. CA Cancer J Clin 2003; 53 : 5-26.

2. Labrie F, Candas B, Gomez JL, Cusan L. Can combined androgen blockade provide long-term control or possible cure of localized prostate cancer? Urology 2002; 60: 115-9.

3. Labrie F, Bélanger A, Cusan $L$, et al. Antifertility effects of LHRH agonists in the male. J Androl 1980; 1: 209-28.

4. Huggins C, Hodges CV. Studies of prostatic cancer. I. Effect of castration, estrogen and androgen injections on serum phosphatases in metastatic carcinoma of the prostate. Cancer Res 1941; 1: 293-307.

5. Bolla M, Gonzalez D, Warde $P$, et al. Improved survival in patients with locally advanced prostate cancer treated with radiotherapy and goserelin. $N$ Engl J Med 1997; 337: 295-300.

6. Pilepich MV, Caplan R, Byhardt RW, et al. Phase III trial of androgen suppression using Goserelin in unfavorable prognosis carcinoma of the prostate treated with definitive radiotherapy: report of radiation therapy oncology group protocol 85-31. J Clin Oncol 1997; 15: 1013-21.

7. Messing EM, Manola J, Sarosdy M, Wilding G, Crawford ED, Trump D. Immediate hormonal therapy compared with observation after radical prostatectomy and pelvic lymphadenectomy in men with node-positive prostate cancer. $N$ Engl Med 1999; 341: 1781-8.
8. Labrie F, Candas B, Dupont $A$, et al. Screening decreases prostate cancer death: first analysis of the 1988 Quebec prospective randomized controlled trial. Prostate 1999; 38 : 83-91.

9. Granfors T, Modig H, Damber JE, Tomic R. Combined orchiectomy and external radiotherapy versus radiotherapy alone for nonmetastatic prostate cancer with or without pelvic lymph node involvement: a prospective randomized study. J Urol 1998; 159: 2030-4.

10. Hanks GE, Lu J, Machtay M, et al. RTOG Protocol 92-02: A Phase III trial of the use of long term androgen suppression following neoadjuvant hormonal cytoreduction and radiotherapy in locally advanced carcinoma of the prostate. In: Program and abstracts of the American Society of Clinical Oncology $36^{\text {th }}$ Annual Meeting May, 20-23: New Orleans. Americain Society of Clinical Oncology, 2000 : 1284 (abstract).

11. Labrie C, Bélanger A, Labrie F. Androgenic activity of dehydroepiandrosterone and androstenedione in the rat ventral prostate. Endocrinology 1988; 123 : 1412-7.

12. Labrie F. Intracrinology. Mol Cell Endocrinol 1991; 78: C113-C8.

13. Labrie F, Dupont A, Bélanger A. Complete androgen blockade for the treatment of prostate cancer. In: de Vita VT, Hellman S, Rosenberg SA, eds. Important advances in oncology. Philadelphia: J.B. Lippincott, 1985: 193217.

14. Labrie F, Luu-The V, Lin SX, Simard J, Labrie C. Role of 17beta-hydroxysteroid dehydrogenases in sex steroid formation in peripheral intracrine tissues. Trends Endocrinol Metab 2000; 11: 421-7.
15. Stewart PM, Sheppard MC. Novel aspects of hormone action: intracellular ligand supply and its control by a series of tissue-specific enzymes. Mol Cell Endocrinol 1992; 83: C13-C8.

16. Labrie F, Dupont A, Bélanger $A$, et al. New hormonal therapy in prostatic carcinoma: combined treatment with an LHRH agonist and an antiandrogen. Clin Invest Med 1982; 5: 267-75.

17. Labrie F, Bélanger $A$, Cusan $L$, et al. History of LHRH agonists and combination therapy in prostate cancer. Endocrine-Related Cancer 1996; 3: 243-78.

18. Labrie F. Mechanism of action and pure antiandrogenic properties of flutamide. Cancer 1993; 72: 3816-27.

19. Crawford $\varepsilon D$, Eisenberger MA, McLeod DG, et al. A controlled trial of leuprolide with and without flutamide in prostatic carcinoma. N Engl J Med 1989; 321: 419-24.

20. Janknegt RA, Abbou CC, Bartoletti R, et al. Orchiectomy and nilutamide or placebo as treatment of metastatic prostatic cancer in a multinational double-blind randomized trial. J Urol 1993; 149: 77-83.

21. Dijkman GA, Janknegt RA, Dereijke TM, Debruyne FMJ. Long-term efficacy and safety of nilutamide plus castration in advanced prostate-cancer, and the significance of early prostate specific antigen normalization. J Urol 1997; 158: 160-3.

22. Caubet JF, Tosteson TD, Dong EW, et al. Maximum androgen blockade in advanced prostate cancer: a meta-analysis of published randomized controlled trials using nonsteroidal antiandrogens. Urology 1997; 49: 71-8.
23. Denis LJ, Keuppens F, Smith $\mathrm{PH}$, et al. Maximal androgen blockade: final analysis of EORTC Phase III trial 30853. Eur Urol 1998; 33: 144-51.

24. Bennett $\mathrm{CL}$, Tosteson TD, Schmitt B, Weinberg PD, Ernstoff MS, Ross SD. Maximum androgenblockade with medical or surgical castration in advanced prostate cancer: a meta-analysis of nine published randomized controlled trials and 4128 patients using flutamide. Prostate Cancer Prost Dis 1999; 2: 4-8.

25. Labrie F, Dupont A, Suburu $R$, et al. Serum prostate specific antigen (PSA) as prescreening test for prostate cancer. J Urol 1992 ; 147 : 846-52.

26. Labrie F, Candas B, Cusan L, et al. Diagnosis of advanced or noncurable prostate cancer can be practically eliminated by prostate-specific antigen. Urology 1996; 47 : 212-7.

27. Candas B, Cusan L, Gomez $J$, et al. Evaluation of prostatic specific antigen and digital rectal examination as screening tests for prostate cancer. Prostate $2000 ; 45$ : 19-35.

28. Labrie F. Screening and hormonal therapy of localized prostate cancer show major benefits on survival. Cancer J Sci Am 2000 ; 6 : S182-7.

29. Labrie F. Screening and early hormonal treatment of prostate cancer are accumulating strong evidence and support. Prostate 2000; 43: 215-22.

30. American Urological Association. Board of directors report: early detection of prostate. New York : American Urological Association, 2001. 
31. Makinen T, Tammela TL, Hakama M, et al. Prostate cancer screening within a prostate specific antigen range of 3 to $3.9 \mathrm{ng} / \mathrm{ml}$. : a comparison of digital rectal examination and free prostate specific antigen as supplemental screening tests. J Urol 2001; 166: 1339-42.

32. Schröder FH, van der Maas $P$, Beemsterboer $\mathrm{P}$, et al. Evaluation of the digital rectal examination as a screening test for prostate cancer. J Natl Cancer Inst 1998; 90: 1817-23.

33. Lodding P, Aus G, Bergdahl $S$, et al. Characteristics of screening detected prostate cancer in men 50 to 66 years old with 3 to $4 \mathrm{ng} / \mathrm{ml}$ prostate specific antigen. J Urol 1998; 159: 899-903.

34. Schröder FH, Roobol-Bouts $M$, Vis AN, van der Kwast T, Kranse R. Prostate-specific antigen-based early detection of prostate cancer-validation of screening without rectal examination. Urology 2001; 57: 83-90.
35. Bartsch G, Horninger W, Klocker $\mathrm{H}$, et al. Decrease in prostate cancer mortality following introduction of prostate specific antigen (PSA) screening in the federal state of Tyrol, Austria. AUA Annual Meeting. J Urol 2000; 163: 88 (abstract 387).

36. Hugosson J, Aus G, Lilja H, Lodding P, Pihl CG, Pileblad $\varepsilon$. Prostate specific antigen based biennial screening is sufficient to detect almost all prostate cancers while still curable. J Urol 2003; 169: 1720-3.

37. Labrie F, Cusan L, Gomez JL, Diamond $P$, Bélanger $A$. Long-term neoadjuvant and adjuvant combined androgen blockade is needed for efficacy of treatment in localized prostate cancer. Mol Urol 1997; 1: 253-61.

38. Laverdiere J, Gomez JL, Cusan L, et al. Beneficial effect of combination therapy administered prior and following external beam radiation therapy in localized prostate cancer. Int J Radiat Oncol Biol Phys 1997; 37: 247-52.
39. Swedish Breast Cancer Cooperative Group. Randomized trial of two versus five years of adjuvant Tamoxifen for postmenopausal early stage breast cancer. J Natl Cancer Inst 1996; 88: 1543-9.

40. Neri RO, Monahan MD, Meyer JG, Afonso BA, Tabachnick IA. Biological studies on an antiandrogen (SH 714). Eur J Pharmacol 1967; 1: 438-44.

41. Labrie F, Belanger $A$, Dupont $A$, Emond J, Lacoursiere Y, Monfette G. Combined treatment with LHRH agonist and pure antiandrogen in advanced carcinoma of prostate. Lancet 1984 ; 2 : 1090.
42. Luu-The V, Zhang Y, Poirier D, Labrie F. Characteristics of human types 1, 2 and 3 17 beta-hydroxysteroid dehydrogenase activities: oxidation-reduction and inhibition. J Steroid Biochem Mol Biol 1995; 55 : 581-7.
TIRÉS À PART

F. Labrie 